Cahiers d'études africaines

\title{
Le fond de l'art était rouge
}

Transferts artistiques entre l'ancien bloc socialiste et la République populaire du Congo

Nora Greani

\section{(2) OpenEdition}

\section{Journals}

Édition électronique

URL : http://journals.openedition.org/etudesafricaines/20716

DOI : 10.4000/etudesafricaines.20716

ISSN : $1777-5353$

Éditeur

Éditions de l'EHESS

\section{Édition imprimée}

Date de publication : 1 juillet 2017

Pagination : 379-398

ISBN : 978-2-7132-2688-5

ISSN : 0008-0055

\section{Référence électronique}

Nora Greani, «Le fond de l'art était rouge », Cahiers d'études africaines [En ligne], 226 | 2017, mis en

ligne le 01 avril 2019, consulté le 02 janvier 2020. URL : http://journals.openedition.org/

etudesafricaines/20716 ; DOI : 10.4000/etudesafricaines.20716 


\section{Le fond de l'art était rouge}

\section{Transferts artistiques entre l'ancien bloc socialiste et la République populaire du Congo}

Le 15 août 1963, soit précisément trois ans jour pour jour après l'accession à l'indépendance de la République du Congo, une révolution populaire désignée plus tard par l'expression "les Trois Glorieuses » ${ }^{1}$ aboutit à la démission, l'emprisonnement puis l'exil du père de l'indépendance, l'abbé Fulbert Youlou $^{2}$. Cette insurrection inaugure dans le pays fraîchement indépendant une nouvelle ligne politique et idéologique orientée vers le socialisme. En voici les principaux jalons historiques. Un laïc, Alphonse Massamba-Débat, succède à l'abbé. En accord avec l'aspiration idéologique de la révolution qui l'a porté au pouvoir, il proclame le socialisme scientifique «doctrine officielle de l'État ». Deux grandes conceptions s'affrontent durant son mandat : celle d'un socialisme modéré dit «bantou » rejetant la lutte des classes et prôné par le Président, et celle d'un socialisme scientifique plus radical, incarné par le Premier ministre Pascal Lissouba. Au mois d'août 1964, à l'image de nombreuses autres nations africaines, un régime de parti unique est instauré, le Mouvement national révolutionnaire (MNR). Les premières organisations de masse apparaissent dans le pays. Quelque temps après, le 31 décembre 1968, le capitaine Marien Ngouabi accède à la présidence à la suite d'un coup d'État. Un an plus tard, il fonde la première République populaire d'Afrique, la République populaire du Congo, ainsi qu'un nouveau parti unique : le Parti congolais du travail (PCT).

Le ralliement officiel de l'État au socialisme scientifique se poursuit de manière tourmentée sous la présidence de Joachim Yhombi-Opango (19771979), puis de Denis Sassou N'Guesso jusqu'en février 1991, date à laquelle

1. L'expression «les Trois Glorieuses » désigne les trois journées d'insurrection des 13,14 et 15 août 1963. Il s'agit d'une référence à la Révolution française des 27, 28 et 29 juillet 1830. Dans le contexte congolais, «les Trois Glorieuses » désignent également la bataille des 26, 27 et 28 août 1940, à Brazzaville, ayant abouti à la victoire des partisans du général de Gaulle sur ceux du gouvernement de Vichy. Cet événement signe le ralliement de l'Afrique équatoriale française (AEF) à la France libre.

2. Je remercie chaleureusement Monique de Saint Martin et Patrice Yengo pour leurs relectures attentives et leurs commentaires fructueux dans le cadre de l'écriture de cet article. 
la Conférence nationale souveraine, porteuse de l'espoir d'une ère de démocratisation, vient clore ce chapitre de l'histoire. Le drapeau national, rouge à la faucille et à la houe, est replié en tant qu'emblème national. Le pays, dont la transition vers l'économie capitaliste avait déjà été largement amorcée, renoue symboliquement avec le statut de République du Congo, désormais multipartite ${ }^{3}$.

L'étalement sur trois décennies de la politique socialiste de la République du Congo (puis de la République populaire du Congo) masque de profondes évolutions. Le pays relève certes de l'idéologie afro-communiste (Young 1982), mais selon trois phases successives : la première, l'instauration du socialisme sous l'ère du MNR (1964-1968); la seconde, la consolidation et la radicalisation sous la présidence de Marien Ngouabi (1968-1977) ; la troisième, le déclin idéologique jusqu'en 1991, durant lequel les objectifs initiaux sont rompus, mais les apparences sauvegardées. Tout au long de cette période, émergent des œuvres d'art (fresques, statuaires, affiches, timbres, peintures sur toile, etc.) caractérisées par une esthétique spécifique glorifiant le parti unique - le MNR puis le PCT — et l'idéologie révolutionnaire. Ce courant artistique que nous désignons, faute de terme existant jusque-là pour le qualifier, par l'expression «art socialiste congolais » est largement soumis au séquençage temporel décrit supra. La tendance générale actuelle au CongoBrazzaville n'est pas, loin s'en faut, à la nostalgie de ce passé socialiste. Dans la capitale, quelques rares marqueurs culturels ont certes été conservés (lorsqu'ils n'ont pas été détruits durant la guerre civile), mais l'engouement mémoriel contemporain trouve largement ses sources en dehors de la chronologie socialiste 4 . Dès lors, les vestiges de l'imagerie socialiste qui ont résisté au temps fonctionnent comme un aide-mémoire efficace et permettent de renouer avec cette époque où, pour détourner le titre du célèbre documentaire de Chris Marker (1977), le fond de l'art était rouge 5 .

Le nouveau vocabulaire visuel socialiste et révolutionnaire qui apparaît soudainement peu après l'indépendance, constitue un emprunt généralisé à une esthétique marxiste développée et théorisée hors du continent, en URSS surtout, et ce depuis plusieurs décennies. La diffusion de l'imagerie de propagande à travers le monde, la confrontation directe de certains artistes

3. Le début des années 1990 inaugure en fait une longue période de crise politique, sociale et économique. L'ère de la démocratisation réclamée d'une seule voix lors de la Conférence nationale dégénère rapidement en un déploiement de violences sans précédent (YENGO 2006a).

4. En témoigne, par exemple, l'édification du Mausolée Savorgnan de Brazza, hommage au premier colonisateur du pays, inauguré en 2006 sur le bord du fleuve Congo (BAZENGuissa-Ganga 1997 ; Bernault 2010 ; Greani 2016a ; Martin Granel 2010; TONDA 2010).

5. Le titre original du documentaire de Chris MARKER est Le fond de l'air est rouge (1977). Le journal Le Monde diplomatique a réemployé cette formule au passé en intitulant un hors-série paru en 2010 : «1960-1975, Quand le fond de l'air était rouge ». En anglais, le film porte le titre A Grin Without a Cat en référence à Alice aux Pays des merveilles. 
congolais avec la production artistique élaborée dans les pays « frères », les passages, brefs mais mémorables du «Che » à Brazzaville, qui contribuent à diffuser ses « attributs » socialistes (béret kaki, costume militaire, bottes) ${ }^{6}$, sont autant d'éléments d'influence extérieurs à l'origine d'une iconographie socialiste originale, spécifiquement «congolaise». Tout en posant un premier regard sur l'art socialiste, cet article interroge la manière dont l'esthétique socialiste congolaise ainsi que les nouvelles modalités d'organisation de la sphère culturelle, étaient sous-tendues par des transferts artistiques (artistes, œuvres, techniques, vocabulaires visuels ou concepts) résultant de logiques politico-diplomatiques propres à la période de la Guerre froide.

\section{Émergence et établissement d'un art de parti}

Au Congo-Kinshasa, l'apparition de la peinture moderne est fixée à la seconde moitié des années 1920, au moment d'une «révélation» esthétique pour des peintures sur murs de cases (qui en annonce beaucoup d'autres) de l'administrateur colonial belge Georges Thiry. Il n'existe nulle anecdote fondatrice de l'art moderne du même type, rive droite du fleuve Congo. Les premiers de ceux qui s'installèrent à Brazzaville en tant que professionnels indépendants, au cours des années 1940, le firent de manière autonome. Or, selon le Français Pierre Lods, établi à Brazzaville à partir de 1949, les œuvres (paysages et portraits) réalisées dans les ateliers indépendants du pionnier camerounais Gaspard de Mouko et des peintres Guy-Léon Fylla, Jean Balou, Faustin Kistba ou Eugène Malonga, sont insuffisamment révélatrices de leur « congolité ». Aussi, Pierre Lods fonde en 1951 un Centre d'art dans le quartier de Poto-Poto. Suivant une tendance récurrente à l'échelle du continent africain à la même époque, cette structure est destinée à favoriser l'émergence d'une prétendue authenticité noire, sorte d'essence de l'âme africaine, chez de jeunes artistes travaillant sans modèle (Greani 2012). À l'instar de l'École des arts fondée en 1953 à Brazzaville ${ }^{7}$, son financement émane de bourses gouvernementales françaises.

Après l'indépendance, la politique culturelle congolaise se réinvente sans la supervision coloniale. Son financement devient secondaire par rapport à celui de multiples secteurs comme la santé et l'éducation, sauf dans le cas où la production artistique répond au premier grand dessein politique du régime monopartite : la propagande socialiste. La tendance artistique socialiste évolue en parallèle à d'autres formes de créativité puisant leurs sources

6. Ernesto Che Guevara est officiellement reçu à Brazzaville par Alphonse MassambaDébat et Pascal Lissouba en janvier 1965, et s'y rend clandestinement l'année suivante.

7. Fondée deux ans après la création du Centre de Poto-Poto, cette structure aujourd'hui disparue était installée au bord du fleuve Congo à Brazzaville, non loin de la « Case de Gaulle ». Elle se divisait en deux sections : reliure et maroquinerie d'une part, céramique d'autre part. 
dans l'art produit en contexte colonial, tout particulièrement de la main des peintres de Poto-Poto. Il s'agit généralement d'œuvres aux couleurs éclatantes et aux motifs « exotiques » (faune et flore tropicales, activités rurales, instruments de musique, etc.) pouvant indistinctement être qualifiés de congolais, africains ou bantous. Au cours des années 1980, ce type de production artistique se politise pour se muer en un courant artistique spécifique «bantou $»^{8}$. En effet, bien qu'ayant largement amorcé sa transition au capitalisme, le pays est encore une république populaire lorsqu'il s'engage en 1985, à l'initiative du Gabon, dans le projet d'un «Centre international des civilisations bantu » et d'une biennale itinérante d'art bantou (ibid. : 2016b). Peintures et sculptures participent alors au discours du retour à l'authenticité, dans une perspective afrocentriste élaborée par certains disciples de Cheikh Anta Diop, en particulier Théophile Obenga (Fauvelle-Aymar, Chrétien \& Perrot 2000 ; Fauvelle-Aymar 2002).

Au gré des circonstances et de leurs besoins, les artistes pouvaient librement naviguer entre les courants artistiques simultanément représentés sur la scène locale (jusqu'à ce que la Conférence nationale, en 1991, vienne signer la fin de l'art socialiste).

Art et État n'ont jamais été aussi ostensiblement liés dans l'histoire de l'art nationale que sous l'ère du РСT, tandis que ce parti fonctionnait comme parti unique. La production d'un art «officiel», élaboré selon les besoins idéologiques du parti, apparaît cependant quelques années plus tôt, sous l'ère du MNR. Dès cette époque, l'État introduit à l'échelle nationale un « matériel idéologique » selon l'expression d'Antonio Gramsci (1975), autrement dit des instruments techniques de diffusion de l'idéologie. Parmi ceux-ci, figurent la radio, la presse et les stages de formation organisés à l'étranger à l'attention des principaux animateurs idéologiques du pays (Bonnafé 1968 : 359). L'art visuel n'est pas envisagé par l'auteur bien qu'il corresponde également à un puissant vecteur idéologique. Des productions artistiques, œuvres d'art mais aussi pièces de théâtre visant à la propagande du premier parti unique, sont élaborées à partir de la seconde moitié des années 1960, mais le renversement d'Alphonse Massamba-Débat et la création d'un nouveau parti unique rendent caduques les œuvres affiliées au MNR et expliquent leur très rare conservation ${ }^{9}$. Au fil des ans, l'État élargit ses prérogatives (jusque-là limitées à la commande) et investit la totalité de la filière de production artistique,

8. L'art bantou fait référence à un concept ethnique flou qui regrouperait près de cent cinquante millions d'individus, répartis dans une vingtaine d'États d'Afrique. Pour une analyse critique de ce concept, voir J.-P. CHRÉTIEN (1985). De fait, l'ensemble des artistes plasticiens congolais étaient considérés comme appartenant à l'ethnie bantoue à l'époque, socialistes y compris. Les étiquettes «bantoue » et «socialiste» caractérisent deux courants artistiques animés par des idéologies distinctes, respectivement le retour à l'authenticité et la victoire du prolétariat.

9. Le Musée national à Brazzaville, actuellement fermé au public et faisant surtout office de lieu de stockage, conserve encore quelques pièces propres à cette époque. 
depuis la révélation de tempéraments artistiques, la prise en charge de leur formation, leur encouragement, jusqu'à l'acquisition des œuvres d'art et leur présentation à un large public. La propagande du Parti s'organise. Elle se manifeste dans tous les formats, du gigantesque au minuscule, au travers de la création de fresques, de statues, de toiles, d'affiches ou de timbres-poste, généralement commandés dans le cadre de célébrations nationales (fêtes du Parti par exemple). De nouveaux édifices sont également construits et nécessitent d'être décorés, comme le Palais des Congrès à Brazzaville (devenu Palais du Parlement), inauguré en 1984.

L'engagement des peintres en faveur du MNR à un moment donné de leur carrière, n'empêche pas qu'ils jouent ensuite un rôle actif au sein du PCT. Les bouleversements des années 1968-1969 entraînant un renouvellement du paysage politique n'impliquent pas un renouvellement similaire de la sphère artistique $^{10}$. Loin d'être exclus, les artistes sont « récupérés » par le nouveau pouvoir en place. La majorité des commandes répond alors à l'injonction étatique. Les artistes (peintres, sculpteurs, céramistes), pour autant qu'ils acceptent de se soumettre à la ligne idéologique du Parti, trouvent là un marché opportun. Si bien qu'aujourd'hui, dans l'esprit de nombre d'artistes et de professionnels de la culture congolaise que nous avons eu l'occasion d'interroger lors de nos enquêtes, l'ère du PCT résonne encore comme un «Âge d'or» du mécénat étatique, et contraste avec la situation actuelle où des artisans nord-coréens ont été récemment choisis au détriment des artistes locaux pour élaborer les Monuments du septennat ${ }^{11}$.

L'interventionnisme du Parti-État auprès des artistes se concrétise par l'organisation d'expositions et de voyages dans les pays «frères », c'est-àdire les républiques socialistes, au point qu'il n'est pas rare aujourd'hui de rencontrer un artiste congolais possédant quelques notions de chinois ou s'exprimant parfaitement en russe. En 1966, le céramiste Albert Massamba bénéficie d'une bourse d'étude en Chine: «J'avais obtenu une bourse de l'État et je suis parti apprendre le dessin textile à Pékin $»^{12}$.

La même année, le peintre Jacques Iloki ${ }^{13}$ obtient quant à lui une bourse d'étude pour l'URSS et suit durant six ans l'enseignement de l'École supérieure

10. Certains, comme l'écrivain et homme politique Henri Lopès, poursuivent leur carrière politique en dépit des changements de gouvernements. Pour une étude détaillée des acteurs politiques en République du Congo, voir R. BAZENGUISSAGANGA (1997).

11. Les Monuments du septennat (en référence au mandat présidentiel de Denis Sassou N'Guesso de 2002 à 2009) constituent une récente commande groupée de l'État à une compagnie d'exploitation nord-coréenne en vue du réaménagement et de la modernisation du territoire. Édifiés sur les grandes places publiques de la capitale dans le cadre des festivités du cinquantenaire de l'indépendance, ils se composent d'un obélisque, d'une colonne de l'indépendance, d'une statue de la liberté, d'une colombe de la paix et des statues de Fulbert Youlou et de Jacques Opangault.

12. Entretien avec Albert Massamba, janvier 2012, Brazzaville.

13. Né en 1962 à Brazzaville, il est fils de François Iloki et neveu de Nicolas Ondongo, deux piliers et pionniers de l'École de peinture de Poto-Poto. Il s'inscrit dans cette structure en 1984. 
des arts appliqués de Moscou. Comme dans le Congo voisin, ce type de formation artistique à l'étranger préparait les artistes aux applications industrielles de leur art, les dessins de motifs de pagnes par exemple. L'écrivain et ancien ministre Jean-Baptiste Tati Loutard $(2003: 214)$ raconte à propos du voyage en Chine en 1964 de l'un des tout premiers peintres indépendants Guy-Léon Fylla, que ce fut pour lui « l'occasion de voir de près l'art chinois contemporain, essentiellement marqué par la lutte pour l'édification du communisme suivant la pensée de Mao-Tsé-Toung ». En 1987, Fylla participe à un stage d'animation culturelle musique et peinture en RDA. Ces diverses expériences de formation des artistes à l'étranger, financées par le gouvernement, ont contribué à l'accélération de la diffusion de l'imagerie socialiste. Elles participaient également du brouillage entre art et politique, l'artiste voyageant souvent dans le cadre de délégations gouvernementales. Ainsi, lors de son second séjour d'études à Pékin en 1984, Fylla est membre d'une délégation de six représentants nationaux, choisis par les autorités congolaises - l'actuel Président récemment réélu Denis Sassou N'Guesso, alors fonctionnaire d'État, fait partie de cette délégation (Fylla 2010 : 42). Il suit sa formation en qualité de peintre, mais aussi de secrétaire exécutif à l'organisation de l'Union nationale des écrivains et artistes congolais.

Les prix décernés aux différents artistes renseignent également sur la politisation généralisée de la sphère culturelle. Émile Mokoko se voit par exemple décerner par l'État le « Premier prix international du centenaire de Lénine », le «Premier prix international Che Guevara» ainsi que le «Premier prix anniversaire de la Chine », tandis que Sylvestre Mangouandza est honoré en 1985 d'une médaille du ministère de la Culture de la République populaire de Chine. Ces prix, ces touches de rouge pourrait-on dire, dans les curriculum vitae des artistes, constituent de saisissants indices géopolitiques, propres à la répartition bipolaire mondiale au temps de la Guerre froide.

\section{Des artistes unis et engagés}

L'expression artistique socialiste se développe essentiellement dans le cadre urbain (Brazzaville et Pointe-Noire) où la proximité du pouvoir facilite l'accès aux commandes. La politique culturelle vise essentiellement les « masses »: le «peuple », plus que d'éventuels «clients » qu'il convient d'éduquer et de rallier politiquement. Le plus souvent, il n'est nul besoin de savoir lire et écrire, d'avoir suivi de longues études ou d'être féru d'art pour saisir le sens des images qui s'offrent, voire s'imposent au regard. Ainsi, l'arche de la «Fresque de l'Afrique » (1970) est édifiée à l'entrée d'un grand marché couvert du centre-ville désormais démoli. Dans la même veine, de nombreux panneaux peints à la main représentant les grandes figures du socialisme sont installés dans les avenues fréquentées des grandes villes (Brazzaville, Pointe Noire, Loubomo, Nkayi) ou à l'aéroport Maya-Maya à Brazzaville, lieu stratégique d'ouverture internationale. 
Si le contenu des œuvres socialistes milite pour la victoire du prolétariat, le fonctionnement de la politique culturelle s'oriente également vers un idéal de solidarité prolétarienne, comme en témoigne l'Union nationale des écrivains et artistes congolais, ou UNEAC, comité de promotion et de défense des intérêts de l'ensemble des créateurs. Organisation de masse, l'UNEAC est créée en novembre 1978, peu après l'assassinat de Marien Ngouabi, et devient le cadre par excellence de la diffusion culturelle du socialisme. La JMNR (Jeunesse du mouvement national de la révolution) créée au mois d'août 1964, la CSC (Confédération syndicale congolaise) fondée au mois de novembre 1964 et l'URFC (Union révolutionnaire des femmes du Congo) fondée en mars 1965 sont les trois organisations pionnières.

Une autre UNEAC (Unión Nacional de Escritores y Artistas de Cuba), fondée quant à elle à La Havane en 1961 par le poète Nicolas Guillén, constitue un modèle organisationnel. Mais c'est surtout l'UAS (Union des artistes soviétiques) qui inspire l'UNEAC congolaise. Fondée à Moscou en avril 1932, dans le prolongement de l'AKRR (Association des artistes de la Russie révolutionnaire), l'UAS constitue un puissant instrument de contrôle idéologique voué à la célébration du prolétariat (toute autre association est alors dissoute). Elle impose une dépendance des artistes vis-à-vis de l'État, seul acheteur d'œuvres et fournisseur de matériaux de productions artistiques. La tutelle exercée restreint la liberté de création et pousse de nombreux artistes à l'exil. L'adhésion à l'UAS exige de multiples conditions, en particulier un ralliement idéologique des artistes. Ceux-ci y trouvent en contrepartie le moyen de vivre de leurs productions plastiques.

En tant qu'organisation de masse, l'UNEAC répond à une volonté politique d'encadrement strict des différentes strates de la population congolaise. Quatre fédérations distinctes répartissent les nombreux adhérents : celles des musiciens, des écrivains, des artistes de scène et, enfin, celle des artistes plasticiens appelée l'UAPC (Union des artistes plasticiens congolais). Cette dernière officialise une tendance générale à la réunion des artistes au sein d'associations ou de groupes de travail (notamment la célèbre École de peinture de Poto-Poto). Elle occupe une position d'intermédiaire, d'une part, pour les hauts responsables de l'État qui peuvent choisir dans le vivier d'adhérents les artistes plasticiens à même d'assurer sa propagande et, d'autre part, pour les artistes qui y trouvent une voie d'accès privilégiée aux commandes officielles. L'ampleur de la plupart des commandes les conduit d'ailleurs souvent à travailler collectivement sur un même projet artistique. Ainsi, la « Fresque de la Révolution » (du nom que nous attribuons à l'œuvre) installée à l'intérieur du Palais des Congrès en 1984, ne réunit pas moins de quinze peintres. On ne compte qu'une femme dans ce corps d'artistes, Laurentine Ngampika, première femme peintre connue du pays. Cette disproportion ne s'explique pas par une éventuelle mise à l'écart systématique des femmes du domaine politique (par exemple, une organisation de masse avait été créée à la seule 
attention des militantes), mais plutôt par la relation établie dès les années 1940 entre métier d'artiste indépendant et individus de sexe masculin ${ }^{14}$.

Le contexte général, a priori peu propice à l'émergence de personnalités singulières sur la scène artistique nationale, n'empêche pas Michel Hengo, Émile Mokoko ou Albert Massamba de devenir peintres officiels de l'État. En matière de statuaire, Bernard Mouanga Kodia devient le monumentaliste officiel à son retour de Marseille au début des années 1980. En mars 2007, à la mort d'Émile Mokoko, le journal Les Dépêches de Brazzaville imprime : «Sur le plan politique, le Parti congolais du travail vient de perdre un militant de la première heure ${ }^{15}$. Le Secrétaire général du PCT lui-même lui rend hommage au siège du Parti, en présence de responsables politiques. Cet hommage posthume consacre a posteriori la forte intrication des champs politique et esthétique sous l'ère du monopartisme.

Contrairement à l'UAS en URSS, entièrement fondée sur le Réalisme socialiste, l'UAPC ne repose pas sur un seul courant esthétique. Les artistes affiliés à cette union ne sont pas sommés de restreindre leurs productions à de l'art socialiste à proprement parler. Qu'ils aient ou non choisi la voie de l'art officiel, tous les artistes doivent apporter une contribution individuelle à cet organisme. Ainsi, lorsqu'une œuvre trouve preneur, notamment au sein de la Galerie centrale prévue à cet effet, un pourcentage du prix de vente est automatiquement prélevé au bénéfice du fonctionnement de l'UNEAC. La plupart des artistes peintres et sculpteurs sont affiliés à l'UNEAC, mais l' «encartement » n'est pas obligatoire. Un faible nombre d'entre eux, principalement représentés par la figure du peintre Hilarion Ndinga, s'opposent immédiatement à ce qu'ils perçoivent comme un embrigadement aliénant :

Il fallait entrer dans cette union qui était une union engagée. Alors j'ai dit : «Non, je ne peux pas faire de la peinture engagée. » [...] Il fallait peindre dans l'esprit de louer les œuvres politiques, louer un individu, faire en sorte que tel personnage se retrouve dans votre tableau... tout ça là. Or, pour moi ce n'était pas de la peinture ${ }^{16}$.

Bien qu'il soit réticent à se voir intégrer au sein d'une organisation à la connotation politique particulièrement forte, Hilarion Ndinga entreprend de son côté la création d'une mutuelle des artistes, conçue comme apolitique, afin d'augmenter les chances d'obtenir des commandes et des soutiens d'ordre financier. En effet, le risque d'une marginalisation sociale et les conditions économiques rendent nécessaire une solidarité d'ordre corporatiste. L'affiliation massive des artistes plasticiens au sein de l'UAPC ne trouve donc pas sa seule explication dans leur ralliement idéologique : «Il fallait

14. Une évolution ne se fera sentir qu'à partir de la seconde moitié des années 1980 et s'officialisera avec l'émergence de la plasticienne Bill Kouélany sur la scène internationale.

15. «Décès de Émile Mokoko», Les Dépêches de Brazzaville, 15/03/2007.

16. Entretien avec Hilarion Ndinga, octobre 2010, Brazzaville. 
être uni. C'était important parce que, vous savez, l'union fait la force. On ne peut pas avancer si on est dispersé $»^{17}$.

Il existe une grande symbiose entre l'art socialiste congolais et l'UNEAC puisque, à notre connaissance, aucune œuvre socialiste n'a été créée en dehors de ce cadre de regroupement professionnel. Lorsque l'art socialiste congolais disparaît définitivement avec la fin du monopartisme, c'est également le cas de l'UNEAC, du moins dans sa forme passée. En effet, l'UNEAC existe toujours, mais elle a changé de statut depuis l'entrée dans l'ère du multipartisme pour devenir une organisation non gouvernementale. Aujourd'hui, son manque de moyens financiers ainsi que son moindre impact concret auprès des artistes contemporains tranchent nettement avec l'époque où elle régissait la quasi-totalité de la créativité au niveau national.

\section{Du contrôle étatique à l'autocensure}

Jeannine Verdès-Leroux (1979 : 35-36) propose de voir en l'artiste de parti un individu «ayant renoncé à remplir pleinement sa définition d'artiste comme producteur en première personne de sa propre légitimité, abdiquant tout ce qui est lié à l'autonomie du champ artistique (choix des sujets, des formes, outils spécifiques, droit de sanction spécifique...)». Ces éléments de définition de l'artiste de Parti communiste français trouvent un écho certain chez l'artiste de parti unique socialiste congolais. En effet, la contrepartie de taille attachée par l'État à l'assujettissement idéologique des artistes, et donc à l'obtention de commandes publiques, est une restriction de la liberté artistique selon différentes modalités.

Première commande d'envergure émanant de la sphère politique ${ }^{18}$, la «Fresque de l'Afrique » constitue certainement l'œuvre la plus révélatrice de l'art du PCT. Toujours en place dans le centre-ville de la capitale, elle prend la forme d'une immense arche recouverte de centaines de carreaux de céramique, elle retrace l'histoire du pays depuis la période précoloniale (à la base du monument) jusqu'à l'avènement du socialisme scientifique (au sommet). L'iconographie prolétarienne jumelée à la lutte anticoloniale fait de cette œuvre à la fois une vaste allégorie de libération du continent et un témoignage artistique socialiste.

En haut et à gauche, une figure inscrit sur une banderole les mots « socialisme scientifique » à l'aide d'un pinceau. Sa position, en tête d'une manifestation «musclée », signale que l'outil de prédilection du peintre exprime toute sa force lorsqu'il est à l'œuvre, dans l'action, au même titre que les armes. Le statut des peintres en particulier et des artistes en général au sein du système révolutionnaire congolais est ainsi mis à l'honneur. La signature

17. Entretien avec Cyrille Bokotaka, janvier 2012, Brazzaville.

18. Il s'agit précisément d'une commande de la Direction générale des affaires culturelles. 
de l'œuvre, sur le bord supérieur gauche, «Le peuple parle au peuple » permet en outre de souligner leur mission pédagogique auprès des « masses ».

Michel Hengo signale qu'une fois cette fresque achevée, la signature lui fut interdite ainsi qu'à ses autres «camarades »:

À l'époque du PCT, on faisait des choses qui étaient un peu dictées par les autres. À la fin des travaux, nous on voulait signer. On nous a dit: «Non! Vous ne pouvez pas signer. Il faut mettre : Le peuple parle au peuple. » Ce n'est qu'après, je crois dix ans après, alors qu'il y avait quelques carreaux qui avaient sauté, qu'ils ont fait appel à nous pour reproduire les mêmes carreaux. On a demandé à la direction [Direction générale des affaires culturelles] : «Maintenant les choses ont changé, nous allons aussi signer notre œuvre. » Ils nous ont donné la permission, nous avons signé ${ }^{19}$.

Le renoncement de ces hommes à « remplir pleinement [leur] définition d'artistes » est particulièrement symbolique dans ce cas. Les signatures de Michel Hengo, Émile Mokoko, André Ombala, Jean Itoua et Dégo n'apparaissent sur l'un des carreaux de céramique, en bas à gauche de l'œuvre, que dans un second temps : au moment où devient désuète l'idéologie de l'effacement de l'artiste en tant que simple atome du peuple devant l'œuvre révolutionnaire.

De manière générale, la propagande est fabriquée « de l'intérieur » (par des artistes congolais) et son usage est largement interne (destinée aux Congolais $)^{20}$. Il convient toutefois de relever au moins deux entorses à cette règle. D'une part, certains Occidentaux interviennent ponctuellement dans le processus de création artistique socialiste congolais. Ainsi, la «Fresque de l'Afrique » est réalisée sous la supervision d'un artiste italien surnommé Dégo. D'autre part, la réalisation de timbres d'art est directement liée aux filiales d'impression installées en dehors des frontières nationales. Les imprimeries françaises Delrieu, Edila, mais surtout Cartor, sont chargées d'assurer une prestation irréalisable au niveau local du fait du manque d'équipements. Des artistes congolais réalisent les maquettes de timbres qui sont ensuite envoyées à des professionnels occidentaux, susceptibles de modifier leurs dessins avant l'impression. Le privilège de retouche est ainsi abandonné à un autre artiste.

La position privilégiée de l'État en matière de gestion culturelle pose évidemment la question du contrôle et de la censure de la production artistique, pouvant aller jusqu'au remaniement autoritaire des œuvres. Le rôle de censeur du Parti-État est un fait avéré. Ainsi, une Commission de censure est mise en place par le MNR en 1964 et reprise par le PCT. Ce service dépend du département «Presse et propagande», placé sous la responsabilité du

19. Entretien avec Michel Hengo, décembre 2009, Pointe-Noire.

20. Avec les timbres d'art, la propagande était non plus destinée exclusivement au public national, mais pouvait servir, par l'envoi de courriers timbrés, à la propagation des idéaux du communisme international à travers le monde. 
Fig. 1. - Fresque de L'Afrique, 1970, BrazZaVille. DÉTAILS BORD SUPÉRIEUR GAUCHE

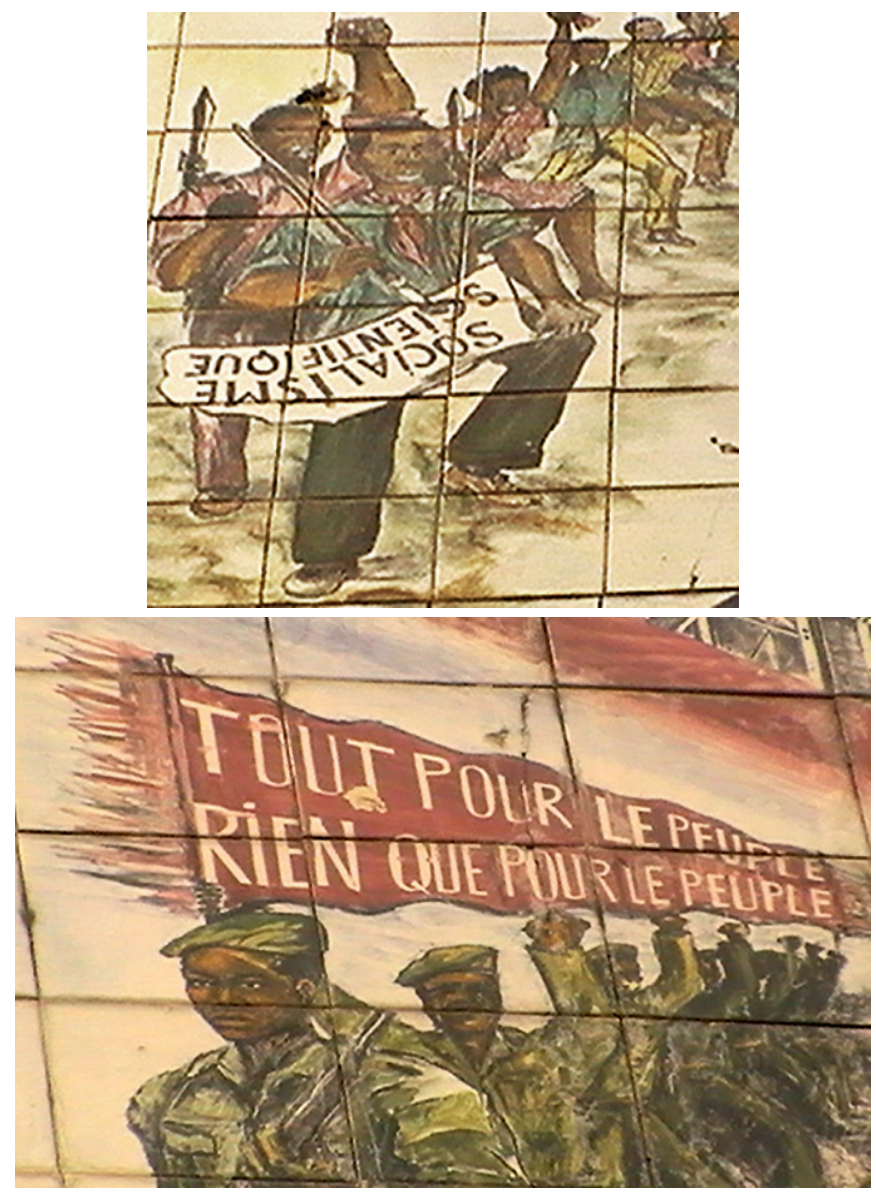

Photographies de Nora Greani, 2013.

ministre de l'Information. Presse et littérature sont tout particulièrement soumises à son contrôle ${ }^{21}$. Plusieurs romans (comme En quête de la liberté de Jean-Pierre Makouta-Mboukouet) ${ }^{22}$ et pièces de théâtre (comme La liberté

21. Plusieurs écrivains célèbres tels que Sony Labou Tansi, Emmanuel Dongala, Maxime N'Débéka ou Guy Menga, ont été publiés à l'étranger (puisque seuls les écrits destinés à la publication locale étaient soumis à une censure préalable). Toutefois, comme nous le précise P. YENGO (2006b), c'est avant tout la capacité des écrivains à se constituer en une sorte de «Société de Lettres» (que son initiateur, Sylvain Bemba, appelait la «phratrie» des écrivains congolais) qui leur permit de déjouer la censure.

22. À propos de la censure de cet ouvrage datant de 1970, voir J.-R. MousahoudJIBoussamba \& S.-G. Doctrové Mouanou (2002). 
des autres de Caya Makhélé) sont interdits ${ }^{23}$. Certains artistes sont contraints à l'exil, comme l'écrivain Guy Menga, voire sont assassinés, comme le musicien Franklin Boukaka en $1972^{24}$, non pas nécessairement en raison du contenu de leurs œuvres, mais plutôt pour leur engagement politique au sein de l'opposition (Yengo 2006b).

L'art visuel ne dépend pas directement de cette Commission de censure, mais est néanmoins soumis à un droit de regard du ministère de la Culture. Par exemple, lorsque la décision est prise d'abandonner l'ancien drapeau aux couleurs panafricaines vert-jaune-rouge et de se doter d'un nouvel emblème national, l'État organise un concours public de maquettes à destination des artistes locaux :

On nous avait demandé si chacun de nous pouvait donner son idée sur le drapeau congolais. Nous étions cinq. On a retenu ma maquette où j'avais dessiné deux palmes croisées en bas, avec des étoiles, la houe et le marteau. On avait les idées sur les pays de l'Est, leur sigle c'était le marteau et la faucille. Je n'ai pas fait la faucille parce que nous, en Afrique, c'était la houe. J'avais placé neuf étoiles qui représentaient neuf régions. Et là, ils ont dit non, c'était un peu trop comme le modèle chinois et on a modifié ça ${ }^{25}$.

Ce témoignage rend compte du rôle actif dévolu aux artistes à l'époque, mais aussi du contrôle exercé par l’État. Par le choix de la houe, Michel Hengo opte pour un recentrement sur les spécificités de la culture congolaise; par le refus des étoiles du drapeau de la République populaire de Chine, l'État entend conduire l'artiste à ne pas imiter à outrance les modèles prétablis dans les autres républiques socialistes.

L'organisation généralisée de concours de maquettes favorise une démarche ambiguë : l'autocensure des artistes. En effet, plutôt que de désigner directement un artiste, l'État confie la réalisation de nombreuses commandes (en particulier les timbres d'art) aux gagnants d'un concours. Pour accroître leurs chances d'être retenus, les artistes se conforment d'eux-mêmes aux attentes supposées du politique. L'intervention étatique n'est pas nécessaire. Proche de celui de la censure moderne, ce mode opératoire se situe «dans le registre de l'implicite, du tacite, en un mot du silence, alors que l'opération de l'interdit appartient au domaine de la parole déclarée » (Valabrega 1967 : 115). L'Etat n'exige pas avec brutalité des artistes plasticiens qu'ils assujettissent leurs pratiques aux directives du Parti. Plus insidieusement, l'essentiel de la sanction encourue par un artiste, s'il ne se

23. Cette pièce a été jouée pour la première fois en 1980 au Théâtre du Centre culturel français de Brazzaville, dans une mise en scène de l'auteur. Sa retransmission à la télévision fut par contre interdite. Lors d'une représentation en direct, un coup de fil du ministère de l'Intérieur aurait intimé l'ordre au directeur des programmes de mettre immédiatement fin à la transmission au bout d'une vingtaine de minutes (CAYA MAKHELÉ $2012: 14$ ).

24. Sur l'une des pochettes de vinyle (label CDI 605, fin des années 1960), Franklin Boukaka pose devant la «Fresque de l'Afrique».

25. Entretien avec Michel Hengo, décembre 2009, Pointe-Noire. 
calque pas suffisamment sur ses exigences, consiste tout simplement en son exclusion du système, synonyme de graves difficultés pour parvenir à vivre de son art.

\section{Un décalage historique et artistique}

Si l'exigence d'un recoupement avec le champ politique avait immédiatement été perçue par les artistes pionniers dans la capitale dès les années 1940 (comme en témoignent les portraits de personnalités politiques, tels ceux du général de Gaulle et du député Jean-Félix Tchikaya réalisés par Gaspard de Mouko), il n'existe aucune tradition plastique socialiste locale. L'apparition de l'art socialiste congolais au début des années 1960 se réalise en décalage avec une large part du reste du monde. Au même moment, les pays dans lesquels le modèle socialiste ou communiste s'est imposé ont déjà développé et théorisé les liens entre art et politique depuis plusieurs décennies. Près de trente ans séparent ainsi les prémices de l'art socialiste congolais des consignes jdanoviennes (du nom de l'idéologue stalinien Andreï Jdanov) qui consacrent le Réalisme socialiste en URSS en 1934.

L'année 1953 signe une détente généralisée en matière de normes artistiques soviétiques. La mort de Joseph Staline et l'arrivée au pouvoir de Nikita Khrouchtchev font souffler un relatif vent de liberté sur la création et autorisent le développement d'un art indépendant, en parallèle de la production artistique officielle. Le modèle du Réalisme socialiste continue de s'exporter, comme en Asie (Cambodge, Républiques populaires de Chine et de Mongolie, Républiques démocratiques populaires de Corée et du Laos, République socialiste du Vietnam), mais les critiques concernant la rigidité de ses canons se multiplient, notamment à Cuba, bastion socialiste attentif à la préservation de la liberté stylistique. C'est durant cette période où la pensée se fait plus critique et moins dogmatique que celle qui triomphait sous l'ère stalinienne qu'apparaît et s'épanouit l'art socialiste congolais. Tandis que les liens avec le bloc de l'Est se renforcent avec la formation de cadres en Bulgarie, en RDA et en URSS, la coopération technique et militaire avec l'URSS et la Chine, l'alignement diplomatique sur la diplomatie soviétique, etc. (Chapiro 2010 : 154), les productions officielles visant à célébrer «à la congolaise » le socialisme scientifique foisonnent.

La volonté des régimes communistes de se prémunir contre le culte de la personnalité n'empêche pas qu'une place centrale soit accordée au chef de parti, cet «interprète des masses » (Labbé 1985). Durant sa première décennie, l'art du PCT se caractérise par une forte diffusion des portraits du Président Marien Ngouabi. Toutefois, sa glorification personnelle n'égale pas celle qui se déploie, par exemple, au même moment au Zaïre autour de Mobutu $^{26}$. Il est instructif d'observer auprès de quelles autres figures politiques emblématiques Marien Ngouabi se trouve représenté dans les œuvres.

26. De nos jours, un culte perdure autour de la figure de Marien Ngouabi comme en témoigne notamment le scintillement (quasi) permanent de la «flamme éternelle» placée à l'entrée de son mausolée dans le centre-ville de Brazzaville. 
Par exemple, au sein de la «Fresque de l'Afrique », son portrait est accompagné de mains tendues, en signe de dévotion (ce qui tranche avec l'athéisme revendiqué par le Parti). Non loin, en haut et à droite, figure le portrait en buste de Patrice Lumumba, héros et martyr de l'indépendance du Congo voisin, mort depuis neuf ans au moment de la création de l'œuvre. D'une part, ce portrait signale l'accueil fait aux différents fronts nationaux de libération cubain, angolais, namibien, camerounais ou encore d'organisations lumumbistes, tel que «Le Mouvement national congolais-Lumumba ». D'autre part, il oppose clairement le gouvernement de Marien Ngouabi au positionnement politique passé de Fulbert Youlou. En effet, le soutien de l'abbé à Joseph Kasa-Vubu (premier Président du Congo-Kinshasa) et Moïse Tschombe (Président de l'État sécessionniste katangais) avait participé à l'isolement politique de Patrice Lumumba, suspecté de sensibilité communiste. La représentation de ce dernier au sein de la «Fresque de l'Afrique » est un symbole fort de sa réhabilitation politique par le régime socialiste.

À l'époque, les avenues et les boulevards sont décorés d'affiches représentant Marien Ngouabi aux côtés de Marx, Engels, Lénine, Che Guevara ou Castro. Des séquences de reportages tournés durant la présidence de Marien Ngouabi montrent que les défilés officiels ne manquent pas de panneaux géants représentant les photographies du Petit père des peuples ou du Grand timonier ${ }^{27}$. Placées côte à côte ou présentées dans un même contexte, ces images produisent un discours légitimateur de la posture politique du Président congolais. Ce type de représentation n'est d'ailleurs pas sans rappeler de nombreuses affiches de propagande socialiste où les visages de Marx, Engels, Lénine, Staline ou Mao sont alignés afin de rendre compte de leur proximité idéologique. La glorification présidentielle trouve également un relai non négligeable avec les timbres d'art. Ainsi, à l'occasion du sixième anniversaire du PCT, en 1975, le peintre Émile Mokoko réalise un timbre en hommage à son principal fondateur. Le Président y apparaît tel un soleil au-dessus de son peuple révolutionnaire, paré de ses attributs habituels : costume kaki et casquette à courte visière, en référence à la fois aux leaders communistes et à son propre statut de militaire. Ces attributs constituent des signes caractéristiques, permettant de le reconnaître immédiatement (à l'instar des soutanes du premier Président par exemple) ${ }^{28}$. L'artiste choisit d'inscrire son visage dans un soleil, ce qui constitue un emprunt caractérisé à l'iconographie maoïste ${ }^{29}$.

27. Le Petit Livre rouge, publié à partir de 1964, atteint le pays et trouve largement ses lecteurs.

28. À l'occasion de la commémoration du septième anniversaire de sa chute en 1970, le Musée national de Brazzaville organisa une exposition critique mettant en scène ses soutanes griffées.

29. Mao incarne dans l'Orient révolutionnaire le «soleil rouge ». Son propre culte de la personnalité se caractérise par d'innombrables œuvres de propagande, dans lesquelles il apparaît entouré de rayons rouges. 
En 1977, l'assassinat de Marien Ngouabi ébranle considérablement l'expérience socialiste congolaise. À partir de la brève présidence de Joachim Yhombi-Opango, suivie de son renversement par Denis Sassou N'Guesso en 1979, le pays s'engage sur la voie d'une transition vers le capitalisme, notamment du fait de l'affaiblissement soviétique, des considérables enjeux pétroliers et du Plan d'ajustement structurel négocié avec le FMI en 1985. L'écho politique se répercute sur les créations artistiques. L'art socialiste congolais continue bien sûr d'exister, mais il entre dans une nouvelle phase : les artistes sont tenus de proposer des œuvres à l'esthétique révolutionnaire qui tenteront de masquer les accointances de plus en plus prononcées du système avec le libéralisme économique. Cette nouvelle orientation coïncide avec la dernière étape du Réalisme socialiste dans les pays soviétiques. En effet, à partir de la fin des années 1970, avec l'ébranlement de la base du pouvoir soviétique affectée par la dégradation des conditions socioéconomiques, les productions artistiques indépendantes et subversives prennent le pas sur la production officielle, «de plus en plus ritualisée » (Carneci 2007 : 10). En République populaire du Congo, la tendance au culte de la personnalité en politique s'atténue peu à peu ou, plutôt, prend une nouvelle forme au travers de la généralisation des photographies et des affiches politiques produites en série qui rendent le recours aux peintres et aux sculpteurs moins nécessaire. Denis Sassou N'Guesso est néanmoins encore abondamment représenté dans les timbres, arborant son béret rouge de paracommando, son foulard également rouge noué autour du cou et son habit militaire kaki.

Le rapprochement entre l'évolution de l'art socialiste congolais et celle du Réalisme socialiste peut être poursuivi, puisque la fin de ces deux

\section{Fig. 2. — « Fresque De L’AfriQue », 1970, BRAZZAVILLE}

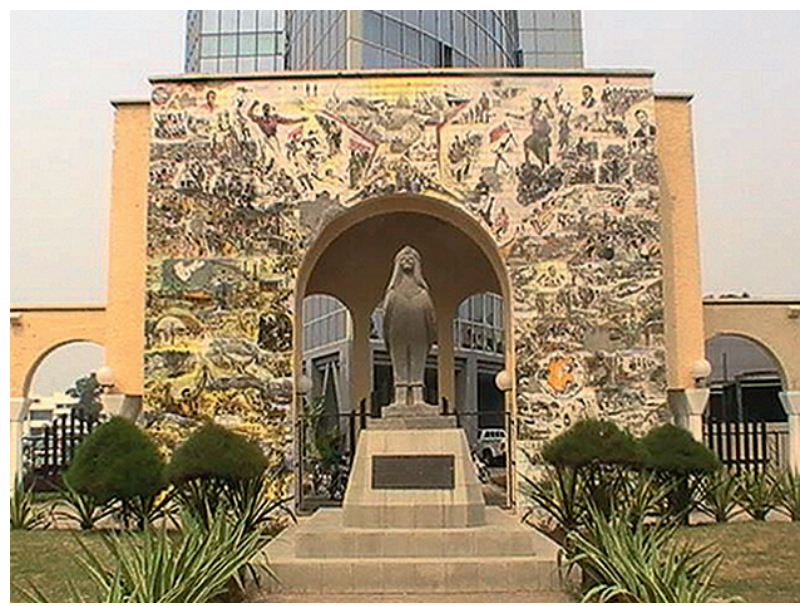

Photographie de Nora Greani, 2013. 
courants coïncide : les années 1989-1990, et 1991 en Afrique répercute le relâchement brutal et hautement perceptible des liens entre art et régimes socialistes et communistes à l'échelle mondiale. La périodisation en deux temps de l'art socialiste congolais s'inscrit globalement dans l'histoire générale du Réalisme socialiste, à une exception notable près : la première étape de ce mouvement artistique, la plus coercitive, ne concerna jamais le CongoBrazzaville alors en situation coloniale. Le balancement original des artistes socialistes congolais entre pratiques codifiées depuis l'étranger et touches de liberté stylistique propres trouve là un facteur d'explication.

En 1991, la crise socio-économique et le contexte géopolitique international contraignent Denis Sassou N'Guesso à convoquer une Conférence nationale. Près de 1200 personnes (syndicats, partis politiques, confessions religieuses, ONG, etc.) participent pendant cinq mois au grand «déballage » retransmis à la radio et à la télévision nationale tandis que des haut-parleurs installés dans la capitale diffusent les débats en direct. La parenthèse de l'art socialiste congolais se clôt au même instant. Plus jamais les artistes n'emploieront l'ensemble de motifs socialistes (codes vestimentaires spécifiques, symboles de la République populaire, postures des figures au poing dressé, etc.). Tous ces signes sont désormais considérés comme appartenant à une époque révolue, que personne ne s'attend à voir réactiver de nos jours.

Parmi les artistes socialistes, certains, comme Rémy Mongo Etsion ou Trigo Piula figurent désormais parmi la génération montante de l'art contemporain congolais (toutes proportions gardées au vu de la spécificité du contexte national en la matière). Les deux artistes les plus connus de nos jours, Marcel Gotène (décédé en 2013) et Bill Kouélany, ne participèrent jamais au courant artistique socialiste. Bill Kouélany ne débute véritablement sa carrière que dans les années 1990, tandis que Marcel Gotène choisit pour sa part de s'exiler en France, dans la Creuse, pour fuir ces bouleversements politiques. Il quitte la République du Congo en 1963 à l'invitation de Jean Lurçat, pour rejoindre la manufacture de tapisserie et sérigraphie d'Aubusson. En proie au «mal du pays », il rentre en 1965, mais, comme il l'explique lui-même :

Hélas, le pays venait d'entrer dans une phase de turbulence « révolutionnaire » avec des changements plus ou moins maîtrisés comme la nationalisation des écoles, l'embrigadement des artistes, le parti unique, le musellement de la presse. Je suis reparti en France et me suis remis à la tapisserie à Aubusson (Gotène 2003 : 12-14).

Il n'a pas existé et n'existe toujours pas de consensus des artistes au sujet de l'ère du «mono », pour reprendre le surnom donné à ce système politique au pays. Beaucoup se souviennent avec nostalgie de cette époque, pour eux synonyme de financement de projets culturels, mais surtout de 
valorisation du statut des artistes à qui la société confiait la charge de la réalisation d'œuvres-phares et de symboles nationaux. De la compétition induite par les concours d'État, ils retiennent surtout l'émulation entre les créateurs ainsi que le sentiment d'appartenance à un groupe. À notre question : «Existait-il une menace, même larvée, qui vous fixait le cadre dans lequel vous pouviez vous exprimer? », le doyen des peintres, Guy-Léon Fylla répond :

Honnêtement, je ne vois pas. Tout ce que j'ai fait, c'était librement. On recevait beaucoup de commandes, beaucoup de peintures. Les artistes n'étaient pas malmenés à cette époque ${ }^{30}$.

Mais d'autres, comme Bill Kouélany, sont bien plus critiques :

Je n'ai pas vu qui a été réellement soutenu au temps du monopartisme comme tu le dis. Et s'il y a des artistes qui allaient de temps en temps dans les bureaux pour qu'on leur donne des enveloppes, ça c'est autre chose, ce n'est pas ça soutenir les artistes $^{31}$.

Le témoignage du directeur du «Cercle culturel Sony Labou Tansi » à Brazzaville, ancien comédien dans la troupe nationale, Prosper Bassaboukila, invite à relativiser le statut de simple exécutant au service du Parti-État des créateurs et même à renverser l'origine de la censure :

C'est vrai que cette politique avait une sorte d'orientation, mais l'artiste, le plasticien, l'écrivain étaient aussi les censeurs du parti. L'artiste avait la possibilité de critiquer, de demander à ce que les responsables de l'État et du parti mènent à bien leurs activités, qu'ils se comportent très bien, qu'ils ne volent pas, qu'ils ne s'acharnent pas sur le bien public. On avait la possibilité de le dire ${ }^{32}$.

Le rôle majeur dévolu à certains artistes (peintres, chanteurs, danseurs, écrivains, comédiens) impliquait selon lui une meilleure audience sur la place publique et autorisait quelques-uns d'entre eux à la critique ouverte. De nos jours, les rares œuvres témoignant encore de l'ère socialiste ont vu leurs significations premières renversées. L'aspect fonctionnel de l'arche de la «Fresque de l'Afrique » a été anéanti au moment du boom pétrolier, lors de la démolition du marché et de son remplacement par un immeuble moderne en verre de onze étages, siège d'une société d'assurance, inauguré en 1980. Une barrière ainsi qu'une sculpture égyptianisante ont définitivement modifié la relation de l'œuvre avec son public, puisqu'il n'est plus possible de la traverser. La statue de bronze en pied de Marien Ngouabi, tombée pendant la guerre civile, a été rénovée durant le mandat de Denis Sassou N'Guesso. On remarquera toutefois que les drapeaux qui désormais flottent au vent derrière cette statue portent les couleurs panafricaines de la République du

30. Entretien avec Guy-Léon Fylla, novembre 2011, Brazzaville.

31. Entretien avec Bill Kouélany, octobre 2010, Brazzaville.

32. Entretien avec Prosper Bassaboukila, octobre 2010, Brazzaville. 
Congo. Le drapeau communiste reste néanmoins partiellement d'actualité puisqu'il constitue l'emblème du PCT dans le contexte multipartite actuel. Lorsqu'en janvier 2016 ce parti investissait le «camarade» Denis Sassou N'Guesso candidat à l'élection présidentielle, ce dernier était acclamé par des centaines de membres du Parti brandissant des drapeaux rouges à la faucille et à la houe. Toutefois, lors de sa campagne électorale ou de son discours d'investiture du 16 avril 2016 faisant suite à sa réélection dès le premier tour le 20 mars 2016, le chef d'État était mis en scène uniquement avec des drapeaux nationaux vert-jaune-rouge. Ainsi, les quelques rares signes témoignant encore de l'héritage communiste du parti au pouvoir sont « réactivés » dans des circonstances politiques locales très particulières, au risque de discréditer le gouvernement sur la scène politique internationale.

Laboratoire d'Anthropologie et d'Histoire de l'Institution de la Culture - Institut interdisciplinaire d'anthropologie du contemporain (LAHIC-IIAC), CNRS-EHESS, Paris.

\section{BIBLIOGRAPHIE}

BAZENGUISSA-GANGA, R.

1997 Les voies du politique au Congo : essai de sociologie historique, Paris, Karthala.

Bernault, F.

2010 «Colonial Bones : The 2006 Burial of Savorgnan de Brazza in the Congo », African Affairs, 109 (436) : 367-390.

BONNAFÉ, P.

1968 «Une classe d'âge politique : la JMNR de la République du Congo-Brazzaville », Cahiers d'études africaines, VIII (3), 31 : 327-368.

CARNECI, M.

2007 Art et pouvoir en Roumanie, 1945-1989, Paris, L'Harmattan.

Chapiro, M.

2010 «République populaire du Congo (1963-1991)», in F. Arzalier (dir.), Expériences socialistes en Afrique, 1960-1990, Paris, Le Temps des cerises: 150-163.

Chrétien, J.-P.

1985 «Les Bantous, de la philologie allemande à l'authenticité africaine », Vingtième Siècle. Revue d'histoire, 8 (8) : 43-66.

Éco, U.

1965 L'œuvre ouverte, Paris, Gallimard. 
FAUVElle-Aymar, F.-X.

2002 « Naissance d'une nation noire. Multimédia, mondialisation et nouvelles solidarités », L'Homme, 161: 75-90.

Fauvelle-Aymar, F.-X., Chrétien, J.-P. \& Perrot, C.-H. (eds.)

2000 Afrocentrismes: l'histoire des Africains entre Égypte et Amérique, Paris, Karthala.

FYLLA, L.-F.

2010 Guy-Léon Fylla, le peintre musicien, Paris, Présence Africaine.

GoTÈNE, M.

2003 Gotène, artiste peintre, Brazzaville, Collection Mémoires.

Gramsci, A.

1975 Quaderni del carcere, vol. 1, Rome, Giulio Einaudi Editore.

GREANI, N.

2012 «Soixante ans de création à l'École de peinture de Poto-Poto », Cahiers d'Études africaines, LII (1), $205: 259-267$.

2016a «Fragments d'histoire congolaise. Les archives coloniales réactivées du Mausolée Savorgnan de Brazza et de la Fresque de l'Afrique », Gradhiva, $24: 82-105$.

2016b «Biennale d'Art Bantu Contemporain : passeport ethnique et circulations artistiques en Afrique sub-sahélienne»,Artl@s Bulletin, 5 (2) : 70-82.

LABBÉ, D.

1985 «Nous les communistes », Mots, 10 : 133-146.

MARKER, C.

2008 [1977] Le fond de l'air est rouge. Scènes de la Troisième Guerre mondiale, film documentaire, $4 \mathrm{~h} .1^{\text {re }}$ partie : Les mains fragiles; $2^{\mathrm{e}}$ partie : Les mains coupées, ISKRA-INA-DOVIDIS.

Martin Granel, N.

2010 «"Abracadabrazza" ou le roman du Mémorial Pierre Savorgnan de Brazza », Cahiers d'Études africaines, L (1), 197 : 293-306.

Mousahoudji-Boussamba, J. R. \& Doctrové Mounau, S. G.

2002 Jean-Pierre Makouta-Mboukou romancier, Paris, L'Harmattan.

TATI LOUTARD, J. B.

2003 Libres mélanges : littérature et destins littéraires, Paris, Présence africaine.

TONDA, J.

2010 «Le Mausolée Brazza, corps mystique de l'État congolais ou corps du "négatif” », Cahiers d'Études africaines, L (2-3-4), 198-199-200 : 799-821.

VALABREGA, J.-P.

1967 «Fondements psycho-politique de la censure », Communications, 9: 114-121. 
Verdes-Leroux, J.

1979 «L'art de parti. Le parti communiste français et ses peintres », Actes de la recherche en sciences sociales, 28 (1) : 33-55.

YENGO, P.

2006a La guerre civile du Congo-Brazzaville, 1993-2002. "Chacun aura sa part», Paris, Karthala.

2006b « À l'ombre de Frantz Fanon: Franklin Boukaka et l'engagement politique en musique congolaise », in H. Maupeu, C. Albert \& A. Kouvouama (dir.), Intellectuels populaires. Un paradoxe créatif, Pau, PUPPA.

Young, C.

1982 Ideology and Development in Africa, New Haven, Yale University Press.

\section{RÉSUMÉ}

Première République populaire d'Afrique, le Congo-Brazzaville se caractérise durant près de trois décennies par une production artistique originale, au service de l'édification de l'État socialiste. L'esthétique socialiste et les nouvelles modalités d'organisation de la sphère culturelle sont sous-tendues par divers transferts artistiques résultant de logiques politico-diplomatiques propres à la période de la Guerre froide : formation des artistes congolais à l'Est, circulation des images de propagande ou encore mise en place d'une organisation de masse propre aux artistes. Tenus d'assurer la propagande du régime, plusieurs artistes participent activement à la structuration de l'État, en élaborant par exemple le drapeau national. Cet article pose un premier regard sur le courant artistique socialiste congolais et questionne l'influence de I'URSS, de Cuba ou de la Chine populaire, sur la mise en place d'un art prolétarien " à la congolaise ».

\section{ABSTRACT}

The Ground of the Air is Red. Artistic Exchanges between the Former Soviet Bloc and the People's Republic of Congo. - The first People's Republic of Africa, CongoBrazzaville, produced original art in service to the building of the Socialist State for nearly three decades. Different artistic transfers, resulting from the political and diplomatic logic proper to the Cold War, underpin Socialist aesthetics and new modes of organization in the cultural sphere: the training of Congolese artists in Eastern Europe, the circulation of propaganda images, and the establishment of a collective (mass) organization especially for artists. Responsible for ensuring the regime's propaganda, several artists actively participate in structuring the State, including, for example designing the national flag. This article takes an initial look at the Congolese socialist artistic movement and investigates the influence of the USSR, Cuba and China on the creation of "Congolese-style" proletarian art.

Mots-clés/Keywords : Congo-Brazzaville, République populaire du Congo, Marien Ngouabi, art socialiste congolais, Réalisme socialiste, socialisme africain/CongoBrazzaville, People's Republic of the Congo, Marien Ngouabi, Congolese Socialist Art, Socialist Realism, African Socialism. 